\title{
Molecular characterization of Pseudomonas sp. isolated from lower respiratory tract infection in HIV and non-HIV population by $16 \mathrm{~S}$ rDNA and ARDRA
}

\author{
C Anitha ${ }^{1,6^{*}}$, Sujatha Kabilan², N Rajinish ${ }^{3}$, A Santhosh Kumar ${ }^{4}$, Padma Krishnan ${ }^{1}$, Illaikiam Rasikan ${ }^{2}$, S Senthilkumar ${ }^{5}$ \\ , S Vincent ${ }^{4}$, S Senthamarai ${ }^{6}$, S Sivasankari ${ }^{6}$, P Gunasekaran ${ }^{2}$, Rajasekharan Sikhamani ${ }^{7}$, M Pushkala $^{8}$
}

From 2nd International Science Symposium on HIV and Infectious Diseases (HIV SCIENCE 2014)

Chennai, India. 30 January - 1 February 2014

\section{Background}

$P$. aeruginosa an important pathogen causing lower respiratory tract infections (LRTI) both in HIV and nonHIV population. Molecular characterization of Pseudomonas spp. helps in better understanding of their clonal distribution among these patient populations. Our study aims to discriminate and generate highly specific fingerprints using 16S-rDNA PCR and amplified ribosomal DNA restriction analysis (ARDRA) techniques.

\section{Methods}

Seventy-two isolates (45-HIV, 24-Non-HIV, 2-environmental, 1-ATCC) of Pseudomonas spp. were subjected to 16SrDNA PCR using universal primers and amplicons digested with Hae III, Alu I and Rsa I for ARDRA analysis. Nucleic acid size confirmation of the digested amplicons was done using MultiNA Bioanalyser. Phylogenetic tree was constructed by maximum parsimonious method using MEGA 4.0. Representative isolates from the major clones were sequenced and submitted to genbank for accession numbers and genetic relatedness was identified by UPGMA using NTSYS 1.80 software. Mean genetic distance (GD) and intraspecies mean GD were calculated.

\section{Results}

Based on ARDRA banding pattern, 14 groups and 10 clones were obtained from the 72 pseudomonas isolates with the following accession numbers: JF279962-64, JF303639-45. Sequences showed 97-100\% similarity with known P. aeruginosa, P.putida, P.stutzeri, Alcaligenes

\footnotetext{
*Correspondence: ani.phd@gmail.com

'Dept of Microbiology (Faculty of Medicine), Dr. ALM PGIBMS, University of Madras, Taramani, Chennai, India

Full list of author information is available at the end of the article
}

feacalis strains by BLASTN analysis. The overall mean GD and intra-species GD of the various species was as follows: P. aeruginosa (JF279962, JF279963, JF303643, JF279964, JF303645, JF303644): 4.664, 16.663, 7.536, 0.733, 0.096, 2.402 and $0.221,3.487,2.010,0.029,2.728,0.011 ;$ P. putida (JF303640, JF303639):11.904, 7.3 and 1.064, 1.371; P. stutzeri (JF303641) is 2.732, 0.036; Alcaligenes feacalis (JF303642) : 30.248, 5.377 respectively.

\section{Conclusion}

The highly variable mean GD values amongst the isolates from different and within the same species indicates high genetic diversity among Pseudomonas spp causing LRTI among HIV and Non HIV patients.

\section{Authors' details}

'Dept of Microbiology (Faculty of Medicine), Dr. ALM PGIBMS, University of Madras, Taramani, Chennai, India. ${ }^{2}$ School of Biological Science, Madurai Kamaraj University, Madurai-21, India. ${ }^{3}$ SRM College, Chennai, India. ${ }^{4}$ LIFE/P.G and Research Department of Advanced Zoology and Biotechnology, Loyola College, Chennai, India. ${ }^{5}$ Dr.K.M.Cherian Heart Foundation and frontier Lifeline Pvt. Ltd, Elavur, Gummidipoondi-601201, India. ${ }^{6}$ Department of Microbiology, Meenakshi Medical College and Research Institute, Enathur, Kanchipuram, India. ${ }^{7}$ Government Hospital of Thoracic Medicine, Tambaram Sanatorium, Tambaram, Chennai, India. ${ }^{8}$ Department of Immunohaemotology, Dr. M.G.R Medical University, Guindy, Chennai, India.

Published: 27 May 2014

\section{doi:10.1186/1471-2334-14-S3-P17}

Cite this article as: Anitha et al: Molecular characterization of Pseudomonas sp. isolated from lower respiratory tract infection in HIV and non-HIV population by 165 rDNA and ARDRA. BMC Infectious Diseases 2014 14(Suppl 3):P17. 